\title{
LHAASO KM2A Muon Detector On-site Test System
}

\section{Shiping Zhao ${ }^{1}$, Yi Zhang ${ }^{1}$, Gang Xiao ${ }^{1}$, Hongliang Dai ${ }^{1}$, Guangguang Xin ${ }^{1,2}$, Xiong Zuo ${ }^{1}$, Zhe Li ${ }^{1}$, Cong Li ${ }^{1}$, Lingyu Wang ${ }^{1}$, Hui Wang ${ }^{1}$, Ning Cheng ${ }^{1}$, on behalf of the LHAASO Collaboration}

${ }^{1}$ Key Laboratory of Particle Astrophysics, Institute of High Energy Physics, Chinese Academy of

Sciences, Beijing, 100049, China

${ }^{2}$ School of Physics and Technology, Wuhan University,

Wuhan, 430072, China

E-mail: zhaospeihep.ac.cn; zhangyieihep.ac.cn

\begin{abstract}
The Muon detector (MD) of LHAASO-KM2A (Large High-Altitude Air Shower Observatory $1 \mathrm{~km}^{2}$ Array) [1] is a water Cherenkov detector with an effective area of $\sim 36 \mathrm{~m}^{2}$. The $2.5 \mathrm{~m}$ thick overburden soil is used as a shield to prevent low energy electromagnetic particles from reaching water. Each MD must be well tested before the covering process of the thick soil. In this case, the on-site test system is designed and manufactured, consisting of a telescope and a data acquisition system. The telescope is a combination of two vertically placed scintillator detectors covered by $4 \mathrm{~cm}$ thick lead each. Charge resolution and time resolution are estimated by analysing the test data. In addition, waveforms of the MD signal are recorded each second, which will serve to evaluate the water quality of MD.
\end{abstract}

36th International Cosmic Ray Conference -ICRC2019-

July 24th - August 1st, 2019

Madison, WI, U.S.A. 


\section{Introduction}

Ground based experiments observe primary cosmic rays (CRs) by detecting secondary particles produced as they interact with atmosphere and develop the extensive air showers (EAS). Generally, more muons will be generated if the primary particle is a hadron rather than a photon or an electron. Thus, measuring the EAS muons would greatly improve the sensitivity for observations of gamma rays. A muon detector (MD) array is therefore proposed in the Large HighAltitude Air Shower Observatory (LHAASO), consisting of 1171 unit muon detectors sampled in an area of about one square kilometers. In July, 2019, a quarter of the MD array has been successfully deployed.

Muon detectors in LHAASO is a water Cherenkov detector with an effective area of $\sim 36$ $\mathrm{m} 2$, shielded by $2.5 \mathrm{~m}$ overburden soil to prevent low energy electromagnetic particles from entering the water and producing backgrounds.[2] However, as long as the soil covering process is completed, it would be difficult to maintain or repair the underground detector. A carefully test must be taken before the covering process to ensure the detector work properly. It is essentially important at the beginning of construction due to the absence of experiences. In addition, performance of the MD is sensitive to water quality, liner reflectivity and photon collection efficiency. Those parameters should be well measured in the test process. In this case, we designed and manufactured a test system, a device which is used to test the MD and to measure essential parameters before the unit detector is buried.

\section{Muon detector}

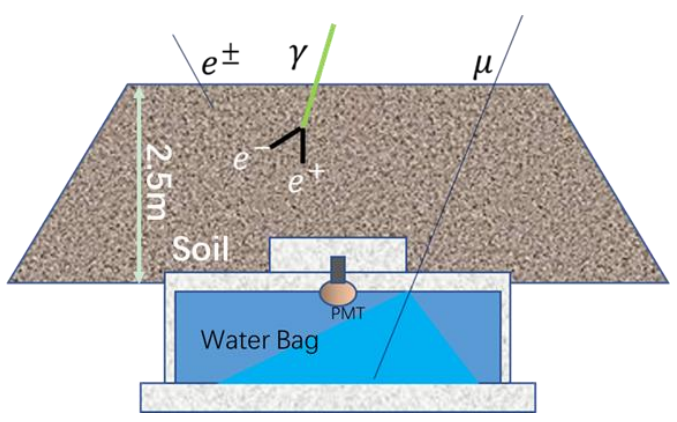

Figure 2.1. Skematic view of a muon detector.

Figure 2.1 shows schematic view of a muon detector. The detector is buried under $2.5 \mathrm{~m}$ of soil (about 18 radiation-lengths) to reduce the punch-through due to the shower electromagnetic particles. In this case, the muon energy threshold is $\sim 1.3 \mathrm{GeV}$. Each cylindrical concrete tank contains a water liner with a diameter of $6.8 \mathrm{~m}$ and a height of $1.2 \mathrm{~m}$ to enclose the pure water. An eight-inch photomultiplier tube (PMT) sits at top center of the liner and looks downwards through a highly transparent window into the water.

The liner provides a 20 -year sealed container for 44 tons of ultra-pure water, protecting the water from contamination and inhibits-by bacteriological activities, as well as a high reflectivity inner surface for the Cherenkov photons. The liner is mechanically supported by the concrete tank and surrounded by the thermal layer.[2] 


\section{On-site test system}

On-site test system consists of a telescope and a data acquisition system (DAQ). The function of the telescope is to filter out low energy particles in showers and provide muons needed by tests.

\subsection{Telescope}

The telescope here is a combination of two vertically placed scintillator detectors, which is shown in figure 3.1. The distance between two scintillators is 80 cm, and each detector has the size of $10 \mathrm{~cm} \times 50 \mathrm{~cm} \times 50 \mathrm{~cm}$. To prevent low energy electromagnetic particles from punching through the both two scintillators, each of the scintillator is covered with a $5 \mathrm{~cm}$ thick lead plate. Events that hit both scintillators will be considered as muon events. In order to estimate the muon purity of selected mensevents, simulations are implemented based on the Geant 4 and CORSIKA package. The proton, as the dominant primary $\mathrm{CR}$, is simulated from $10 \mathrm{GeV}$ to $10 \mathrm{PeV}$, with the energy spectrum index set at 2.7. The air shower is simulated by CORSIKA. At our observation level, the numerous secondary particles are gammas, electrons 3 -/positrons and muons, which are divided into three classes. Energies of them follow the distributions shown in figure 3.2. In Geant4 simulation, these particles are arranged to shoot a $10 \mathrm{~cm}$ thick lead vertically. Probability (in Table 1 it referenced as $\left.P_{\text {penetrate }}\right)$ that a particle penetrating through the lead are calculated. As shown in table 1 , percentages at the second row represent quantity proportions corresponding to three particle classes $\left(\gamma, e^{ \pm}, \mu^{ \pm}\right)$. Thus $2 \sim 87 \%$ of the selected particles in a shower have penetrated through the lead are muons.

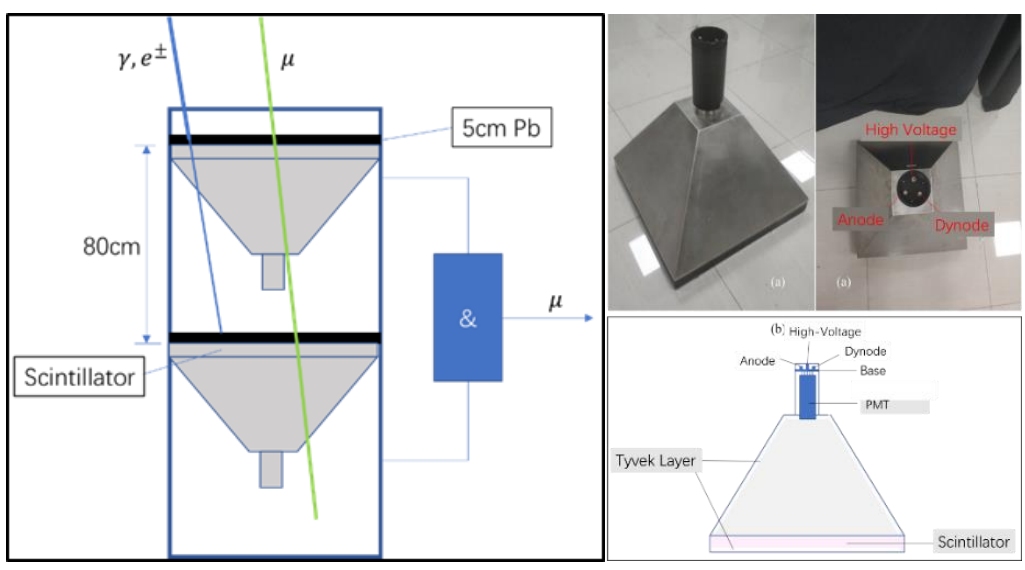

Figure 3.1. Telescope and scintillator detector

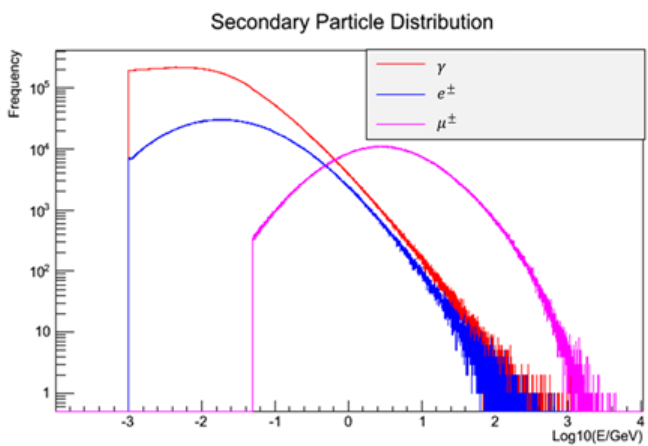


Figure 3.2. Energy distributions of secondary $\underline{\operatorname{secondary}} \gamma, \boldsymbol{e}^{ \pm}, \mu^{ \pm}$in showers with primary $\mathrm{CR}$ energies over $10 \mathrm{GeV}$.

Table 1. Calculation of purity of selected muons.

\begin{tabular}{lccc}
\hline \hline & $\gamma$ & $\boldsymbol{e}^{ \pm}$ & $\boldsymbol{\mu}^{ \pm}$ \\
\hline$\omega(\%)$ & 83.38 & 12.55 & 4.07 \\
$P_{\text {penetrate }}(\%)$ & 0.72 & 0.026 & 99.91 \\
$S(\%)$ & 0.128 & 0.000699 & 0.871 \\
\hline
\end{tabular}

Note: $\omega$ represents the abundance of particles in for the corresponding elassparticles, while $P_{\text {penetrate }}$ is the probability of their particles penetrating through the lead. $s$ represents how much the particles are among those who have penetrated through the lead.

\subsection{DAQ System}

Layout of the DAQ system is shown in figure 3.4. The toppest level is the White Rabbit (WR) switcher which can provide sub-nanosecond synchronization over $1 \mathrm{G}$ Ethernet networks with timing and data transfers among its different elements, which makes all the recorded events clock at the same time.

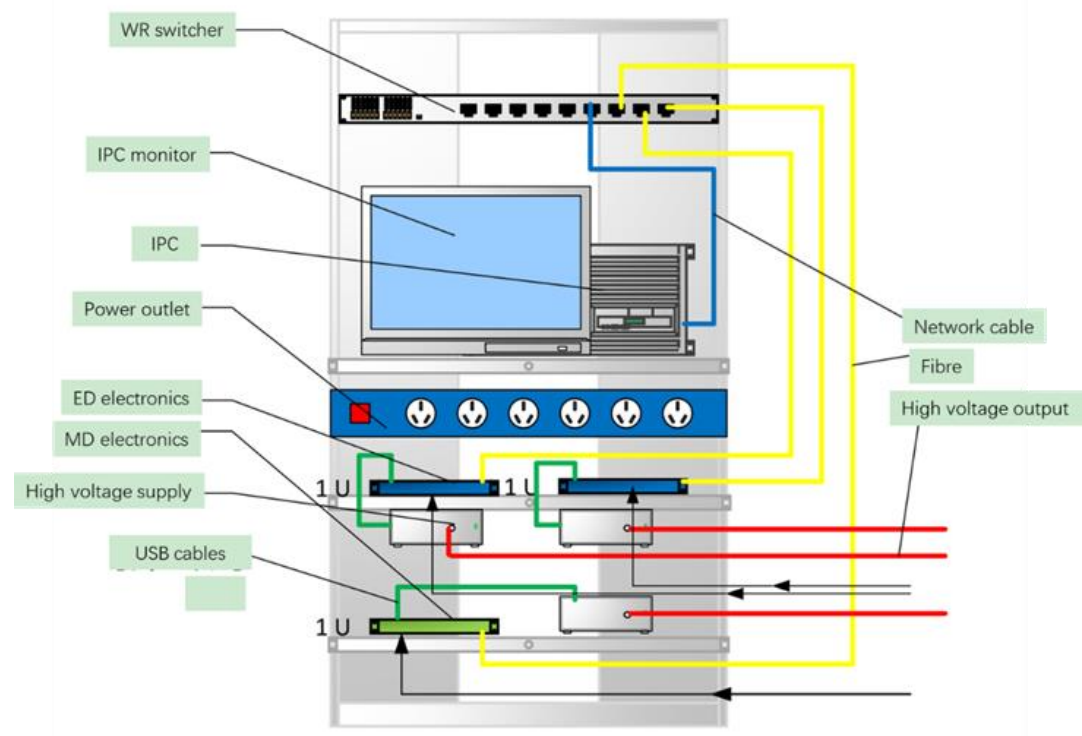

Figure 3.4. Device layout of DAQ cabinet.

The analysis programs are designed and coded on Linux system, controlling the whole progress of the test. Each time when testing the MD, users could edit the configuration information such as thresholds of electronics, IP address, high voltage for PMTs, etc. After data accumulated, parameters such as the number of photoelectrons (NPEs) of PMTs and decay time constant of signals (DTCS) will be calculated. These parameters will be introduced in the next section.

\subsection{NPE and DTCS}

NPE and DTCS are two important parameters of MD.

The number of photoelectrons of PMTs follows a distribution shown in figure 3.5. Fitted by a Gauss function, the mean value is defined as the NPE of a detector. 

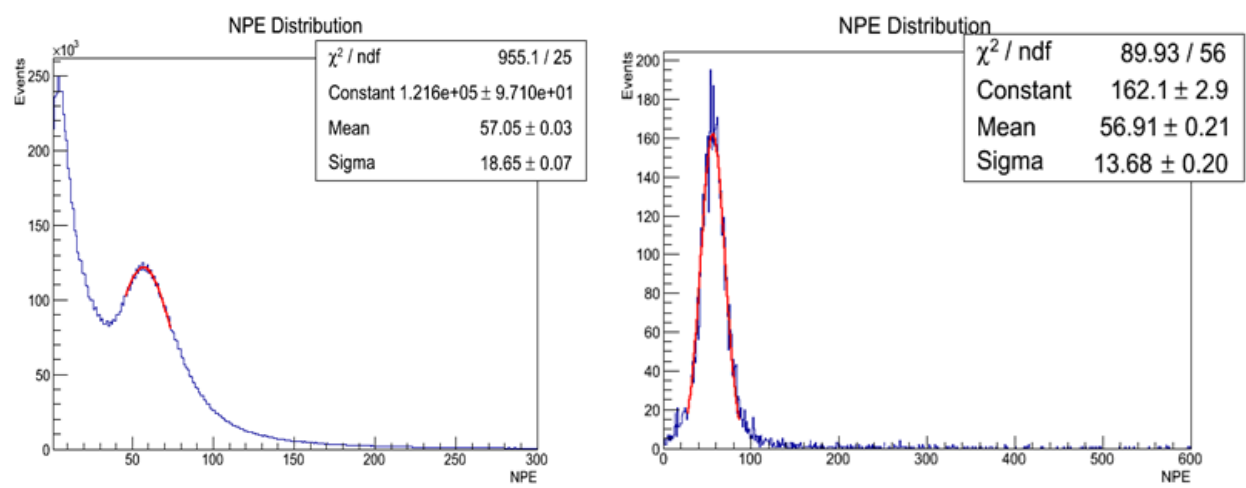

Figure 3.5. NPE distribution before event selection (left) and after event selection (right).

MD signals are exponentially shaped waveforms (see figure 3.6) due to absorption of Cherenkov photons by water and the liner during their transmission. An averaged waveform can be drawn by using waveform data the MD electronics stored, as shown figure 3.7. In this figure the waveform is fitted by an exponential function [3], which is :

$$
C e^{-t / \tau}
$$

in which $\tau$ is the so-called decay time constant, and in figure 3.7 it is $174 \mathrm{~ns}$.

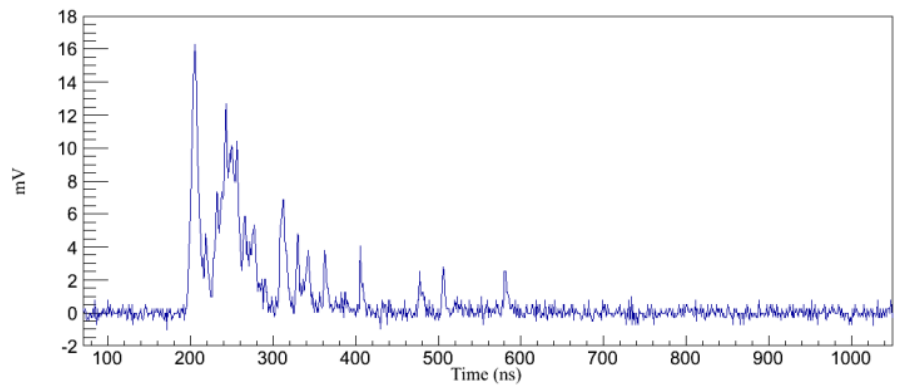

Figure 3.6. An anode signal recorded by electronics.

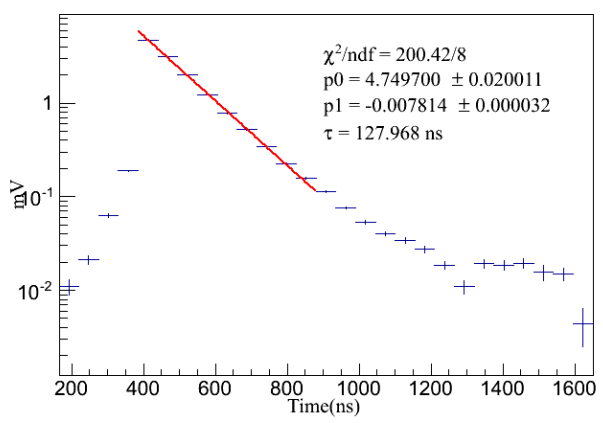

Figure 3.7. Averaged anode waveform fitted by exponential function. Slope here is equal to $1 / \tau$

\section{Results of 50 MDs}

In 2018, 50 MDs, also referenced as first batch MDs, are installed and tested by the test system. Distributions of NPE and DTCS are shown in figure 4.1 and figure 4.2, respectively. From which the good consistency of these MDs is shown. 


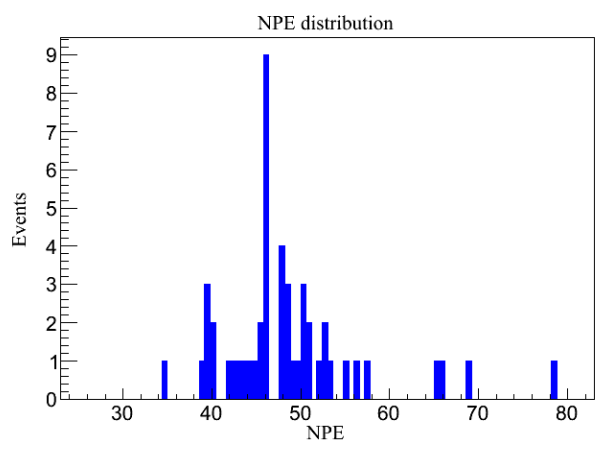

Figure 4.1. 50 MDs' NPE distribution

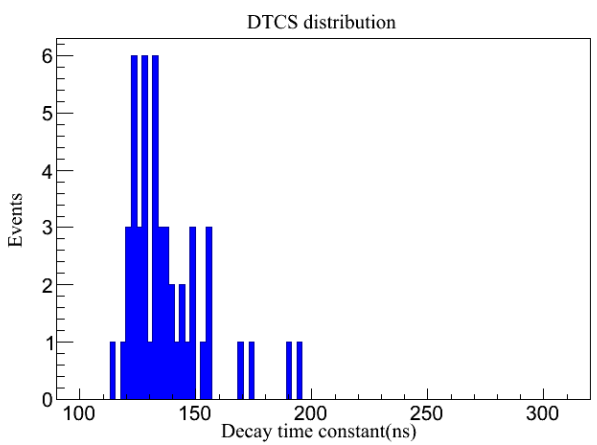

Figure 4.2.50 MDs’ DTCS distribution.

Correlation of NPE and DTCS is revealed in figure 4.3. It shows the sign that a bigger DTCS would corresponds to a bigger NPE, which gives us the suggestion that improving the reflectivity of liner, doubling Tyvek layers for example, would increase the NPE.

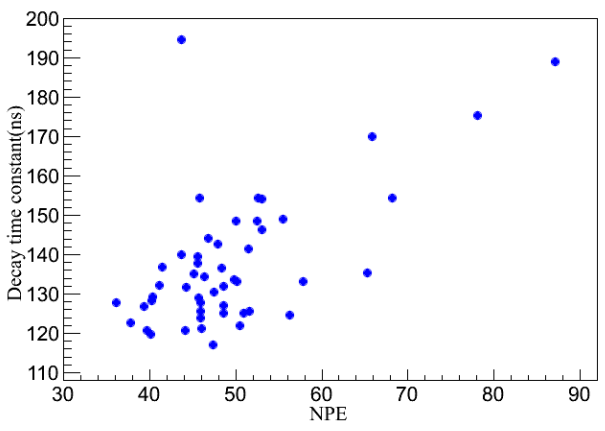

Figure 4.3. Correlation of DTCS and NPE.

\section{Improvements of test system}

In order to increase the efficiency of test work, some simplifications have been made, such as the abandon of telescope, WR switcher and long cables, etc., making the system is as large as a suitcase, as figure 5.1 shown. Unlike the older one, new system can be operated remotely, which gives a lot of convenience to users. 


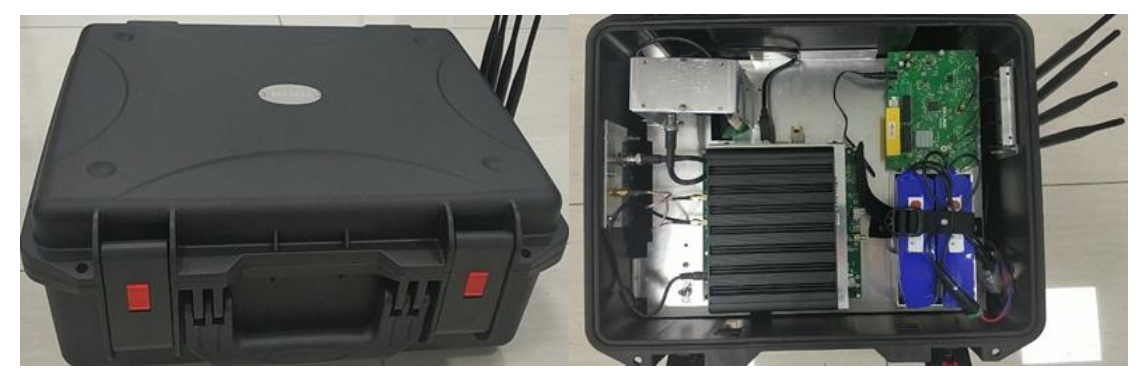

Figure 5.1 The appearance (left) and inside (right) of the new system.

Applying this system, 247 MDs have been installed and tested since April of this year, approaching the goal of completing the quarter array installation. NPE and DTCS distributions of 80 of them are shown in following graphs. Additionally, it shows us in figure 5.4 that MDs with Tyvek layer doubled have bigger NPE and DTCS, which means improving the reflectivity of the liner can indeed increase the NPE or DTCS.

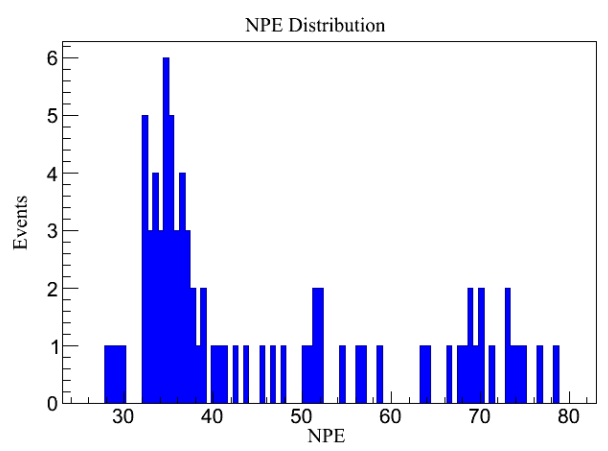

Figure 5.2. NPE distribution of 81 MDs installed in 2019 .

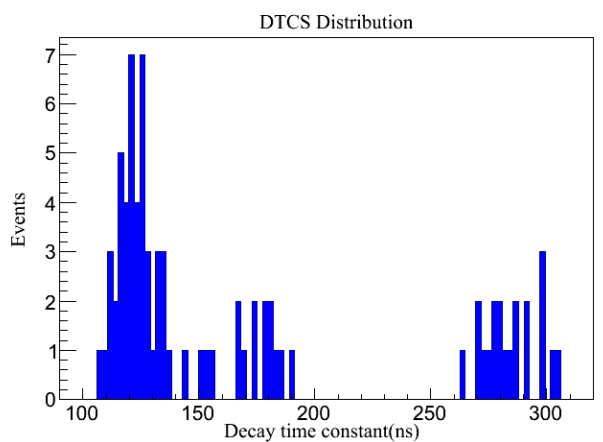

Figure 5.3. DTCS distribution of 81 MDs.

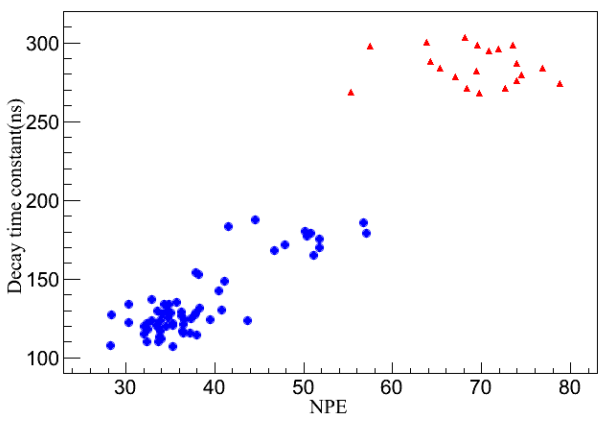


Figure 5.4. Correlation between DTCS and NPE. Rectangle-shaped points in red represent the results of MDs with doubled Tyvek layer, and blue circles corresponding to MDs with single Tyvek layer.

\section{Summary}

An on-site test system was implemented and some improvements have been made for increasing test efficiency. Choosing NPE and DTCS as the parameters to be measured, a MD's performance can be estimated. At the end of 2018, 50 MDs were installed and tested. Results show that most of the MDs are normal and have a good consistency. By applying the improved MDs, more than 274 MDs have been installed and tested since April, 2019. Results of 81 among them are published, from which the fact that MDs which have liner with Tyvek layer doubled get larger NPE and DTCS is revealed. This could be considered as a good example of guidance of improving the performance of MDs.

\section{Acknowlegements}

This work is partly supported by the National Key R\&D Program of China No. 2018 YFA0404202 and National Natural Science Foundation of China under Grant 11775233. Meanwhile, all members in group of KM2A-MD have contributed their time and efforts to installations and tests of MDs, acknolegements here are also for them.

\section{References}

[1] CAO Zheng Chin. Phys. C (HEP \& NP), 2010, 34(2): 249-252

[2] Zuo Xiong, Design and Performances of LHAASO-KM2A Muon Detector (Ph.D. Thesis) Beijing: Institute of High Energy Physics, CAS, 2009 (in Chinese)

[3] Xiurong Li, Huihai He, et al. Study of Water Chrenkov Muon Detector Parameters and Performances for LHAASO, POS (ICRC2015) 571, 2015 